Nathaniel W. Yang, MD

Department of Otorhinolaryngology College of Medicine - Philippine General Hospital University of the Philippines Manila

Department of Otolaryngology Head and Neck Surgery FEU-NRMF Institute of Medicine

\section{Pulsatile Tinnitus Due to a Sigmoid Sinus Diverticulum and/or Dehiscence}

In 2009, a 52-year-old man presented with a two-year history of intermittent right-sided pulse-synchronous tinnitus. He noted that the tinnitus worsened when his blood pressure was elevated. Otologic exam was unremarkable with no obvious middle ear fluid or mass. There was no neck bruit and the tinnitus diminished on manual compression of the ipsilateral internal jugular vein. In keeping with the recommendations for clinical imaging at that time, a non-contrast CT of the temporal bone was performed. This was to evaluate for conditions such as: a middle ear glomus, an aberrant internal carotid artery, a jugular bulb variant (e.g. a highriding jugular bulb), otosclerosis, superior semicircular canal dehiscence syndrome, a persistent stapedial artery, or a hemangioma of the temporal bone.' No evidence of these conditions was found. An MRI of the brain, with MR angiography and venography of the intracranial vasculature also performed to evaluate for conditions such as: idiopathic intracranial hypertension, a dural arteriovenous fistula, an arteriovenous malformation, vascular loop syndrome and dural sinus stenosis or thrombosis. ${ }^{2}$ All of these conditions were excluded. As no definite pathology was identified, no firm treatment recommendations were initiallly made.

In 2011, Eisenman reported on a series of 13 patients with pulsatile tinnitus due to a sigmoid sinus diverticulum and/or dehiscence who were successfully treated surgically via an extraluminal transmastoid approach. ${ }^{3}$ This was the first relatively large series published in the otologic literature. This publication likewise reported on the subtle radiologic signs that signify the presence of a sigmoid sinus diverticulum and/or dehiscence such as an irregularity of the normal semicircular contour of the bony sinus wall, focal thinning of the calvarial cortex overlying the adjacent sinus wall, absence of the normal thin layer of cortical bone overlying the sinus and the "air-on-sinus" sign where mastoid air cells directly contact the sinus wall without overlying bone. ${ }^{3}$

In light of this new information, the patient's imaging studies were re-evaluated and evidence of a right-sided sigmoid sinus diverticulum and/or dehiscence was identified. The images below show the findings on an axial slice of the patient's temporal bone CT study.
Correspondence: Dr. Nathaniel W. Yang

Department of Otorhinolaryngology

University of the Philippines Manila

Taft Avenue, Ermita, Manila 1000

Philippines

Phone: (632) 5264360

Fax: (632) 5255444

Email: nathaniel.w.yang@gmail.com

The author declared that this represents original material, that the manuscript has been read and approved by the author, that the requirements for authorship have been met by the author, and that the author believes that the manuscript represents honest work.

Disclosures: The author signed a disclosure that there are no financial or other (including personal) relationships, intellectual passion, political or religious beliefs, and institutional affiliations that might lead to a conflict of interest.

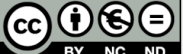




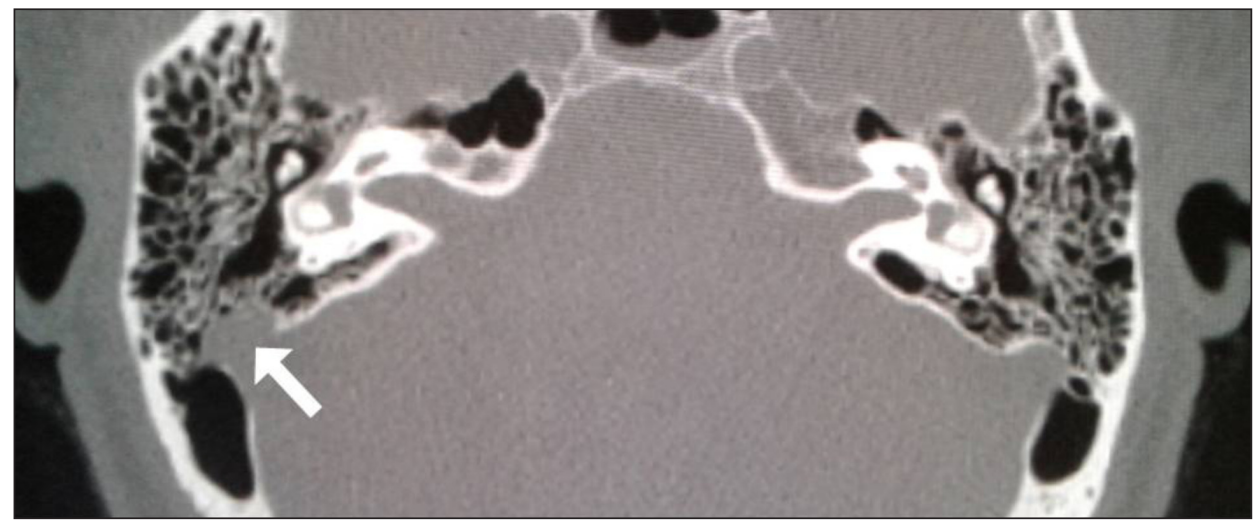

Figure 1. Axial CT image of the temporal bone at the level of the horizontal semicircular canals : absence of the normal thin layer of cortical bone overlying the right sigmoid sinus (white arrow) compared to the left sigmoid sinus

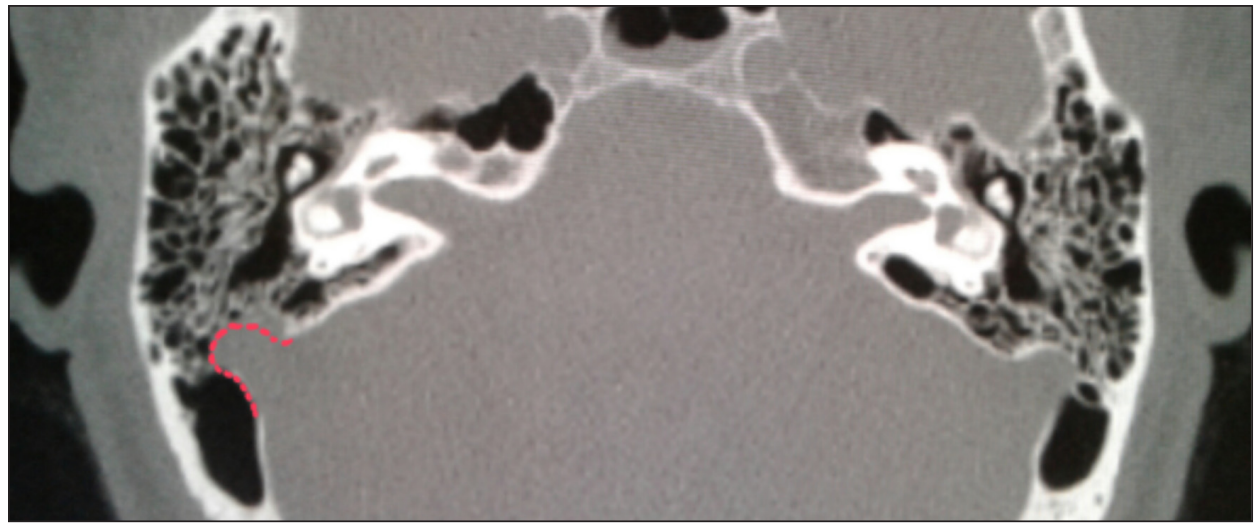

Figure 2. Axial CT of the temporal bones: the location of the sigmoid sinus outlined with the red dotted line, illustrating the irregularity in the normal semicircular contour of the bony sinus wall. A portion of the sigmoid sinus can be seen extending beyond the seeming border of the sigmoid sinus.

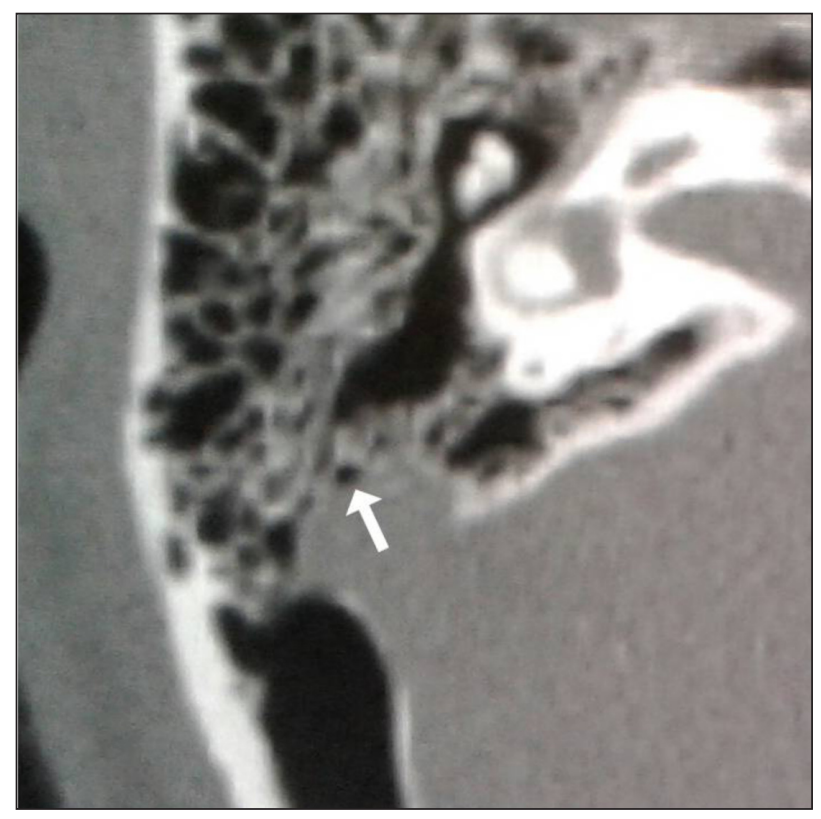

Figure 3. Magnified view of Figure 1 showing the "air-on-sinus" sign, where the mastoid air cell directly contacts the sinus wall, without any overlying bone (white arrow)
How significant is this condition? Sigmoid sinus diverticulum and/ or dehiscence is being increasingly recognized as a common cause of pulsatile tinnitus. In fact, a recent study by Schoeff et al. found its prevalence to be $23 \%$ in patients with pulsatile tinnitus. ${ }^{4}$ As such, the identification of this condition is highly relevant particularly because effective surgical management is available for its alleviation.

\section{REFERENCES}

1. Mattox DE, Hudgins P. Algorithm for evaluation of pulsatile tinnitus. Acta Oto-Laryngologica 2008; 128: 427-431.

2. Shin EJ, Lalwani AK, Dowd CF. Role of angiography in the evaluation of patients with pulsatile tinnitus. Laryngoscope 2000; 110:1916-1920.

3. Eisenman DJ. Sinus wall reconstruction for sigmoid sinus diverticulum and dehiscence: a standardized surgical procedure for a range of radiographic findings. Otol Neurotol 2011; 32 1116-9.

4. Schoeff $S$, Nicholas $B$, Mukherjee $S$, et al. Imaging prevalence of sigmoid sinus dehiscence among patients with and without pulsatile tinnitus. Otolaryngol Head Neck Surg 2014; 150: 841 - 